\title{
TLR2/6 agonists and IFN $\gamma$ synergize to induce melanoma cells to produce T-cell recruiting chemokines
}

\author{
lleana S Mauldin ${ }^{1 *}$, Ena Wang ${ }^{2}$, Donna H Deacon ${ }^{1}$, Craig L Slingluff ${ }^{1}$ \\ From Society for Immunotherapy of Cancer 28th Annual Meeting \\ National Harbor, MD, USA. 8-10 November 2013
}

Melanoma is currently the most lethal form of skin cancer. Clinical efforts to combat melanoma have included approaches to induce the immune system, through T-cell directed therapies, to clear melanoma. These approaches require that immune cells enter the tumor microenvironment to be clinically beneficial. However, most tumors lack significant immune infiltration prior to immune therapy, and immune therapies are hindered by a persistent lack of immune cell infiltration. Chemokines can promote $\mathrm{T}$-cell migration into tumors; therefore agents that induce chemokine production by melanoma cells to promote T-cell infiltration may be clinically beneficial, and could potentially work synergistically with current immune treatments for melanoma. We hypothesized that TLR ligation of melanoma would induce immune cell attracting chemokine secretion directly from melanoma cells, alone or in combination with IFN $\gamma$. To test this hypothesis, we treated human melanoma cell lines (VMM1, DM13, DM93 and DM122) individually with TLR agonists and analyzed effects on the expression of chemokines CCL2-5, CXCL9-10, and CXCL12 by flow cytometric analysis. The following TLR agonists were tested: MALP-2 and FSL-1 (TLR2/6), poly-ICLC (TLR3), LPS (TLR4), CpG (TLR9), Resiquimod (TLR7/8), Imiquimod (TLR7). Our studies found that melanoma express TLRs $2,3,4,6$, 7, and 9, but that without exogenous stimulation, melanoma cells produce few immune cell attracting chemokines. Furthermore, stimulation of melanoma with the TLR agonists alone induced little chemokine production. However, TLR2/6 treatment of melanoma cells (MALP-2 or FSL-1) given in combination with IFN $\gamma$ resulted in significant increases in CXCL10 and CCL3 chemokine production by melanoma. We found that TLR2 and TLR6 are widely expressed on human melanoma cells. Furthermore, stimulation of fresh patient melanoma specimens with TLR2/6 agonists plus IFN $\gamma$ induces CXCL10 production from melanoma cells, endothelial cells and, to a lesser extent, several immune cell subsets including dendritic, monocytic, macrophage, and B-cell. Finally, ex vivo migration assays demonstrated that stimulation of melanoma cells with TLR2/6 agonists plus IFN $\gamma$ increases CD4+ and CD8+ $\mathrm{T}$-cell migration toward melanoma cells. CXCL10 has been implicated as a critical chemokine supporting $\mathrm{T}$-cell infiltration into the tumor microenvironment. Collectively, these data identify TLR2/6 agonists and IFN $\gamma$ as key inducers of CXCL10 production by melanoma cells and suggest that for tumors lacking immune cell infiltrates, systemic immune therapies may be improved by combination with intralesional therapy using TLR $2 / 6$ agonists plus IFN $\gamma$.

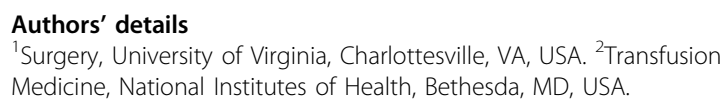

Published: 7 November 2013

doi:10.1186/2051-1426-1-S1-017

Cite this article as: Mauldin et al:: TLR2/6 agonists and IFN $\gamma$ synergize to induce melanoma cells to produce T-cell recruiting chemokines. Journal for ImmunoTherapy of Cancer 2013 1(Suppl 1):017.

${ }^{1}$ Surgery, University of Virginia, Charlottesville, VA, USA

Full list of author information is available at the end of the article

(C) 2013 Mauldin et al; licensee BioMed Central Ltd. This is an Open Access article distributed under the terms of the Creative Commons 